\title{
Two new species of Phanerochaete (Basidiomycotina, Aphyllophorales), and a key to species from subtropical and tropical areas
}

\author{
KURT HJORTSTAM
}

\begin{abstract}
HJORTSTAM, K. 2000: Two new species of Phanerochaete (Basidiomycotina, Aphyllophorales), and a key to species from subtropical and tropical areas. - Karstenia 40: 53-62. ISSN 0435-3402.

Two new species of Phanerochaete are described, viz., P. irpicoides Hjortstam (Brazil) and $P$. tuberculascens Hjortstam (Africa, Burundi). A brief generic description, a checklist and a key to accepted species from subtropical and tropical area are provided.
\end{abstract}

Key words: Aphyllophorales, Basidiomycota, Phanerochaete, tropical species

Kurt Hjortstam, Målaregatan 12, SE-441 35 Alingsås, Sweden

\section{Introduction}

Phanerochaete is still a necessary and convenient generic arrangement for many effused species of corticioid fungi without clamp-connections, whether these species have cystidial elements or not. Undoubtedly it is not a natural group and the genus will certainly be split in the future when DNA tests have been performed. As currently defined, Phanerochaete contains about 70 species, exclusive of Phlebiopsis Jülich (with about 10 species) and Scopuloides (Massee) Hjortstam \& Ryvarden (2-3 species) of which 50 are known from subtropical and tropical areas. Species of Phlebiopsis and Scopuloides have not been included in the key (below). For distribution of these species see Hjortstam and Larsson (1995).

Phanerochaete P. Karst., Bidr. Känned. Finl. Nat. Folk 48: 426, 1889

Generic type: Thelephora velutina DC. : Fr.

Basidiomes generally large and conspicuous, resupinate, sometimes strongly attached to the substratum, but more commonly easily detachable. Hymenophore smooth, though often pilose from protruding cystidia, more rarely ornamented. Colour varying from white to yellow or even reddish or brownish. Some species are discoloured with $\mathrm{KOH}$, turning yellow, green, orange or red. Subiculum generally well developed, mainly whitish or yellowish, rarely red or brownish. Mycelial strands (cords) often present, whitish or yellowish or reddish. Hyphal system monomitic; hyphae without clamp-connections, but often with scattered single or multiple clamp-connections, mainly hyaline or yellowish, rarely brownish, thin-walled or in many cases with thickened walls or firm-walled. Cystidia present or absent, subulate to obtuse, smooth or encrusted, thinwalled or in some cases firm-walled. Basidia more or less clavate, often in a rather dense palisade, with four sterigmata and without a basal clampconnection. Spores smooth, thin-walled, globoid to ellipsoid or cylindric, rarely allantoid, mainly between 4-7 $\mu \mathrm{m}$ long, inamyloid, indextrinoid and acyanophilous.

Phanerochaete irpicoides Hjortstam sp. nov.

Basidioma resupinatum, laxe adnatum, expansum. Hymenophorum valde raduloides, irpicoides vel fere poroides, brunneolum; aculeis dentiformibus, $0.8 \mathrm{~mm}$ altis, quasi $1-2 / \mathrm{mm}$, levibus. Margo indeterminatus, revolubilis. Chor- 
dae nullae. Subiculum aliquantum tenue, hymenophorio concoloro vel plerumque fuscato. Systema hyphale monomiticum; hyphae basales distinctae, crassitunicatae, 5-6 4 m latae, hyalinae, incrustatae; crystalliis subhyalinis. Cystidia sparsa, humile characteristica, tenuitunicata, initio obclavata, plus minus obtusa, raro subcapitata, tum fere subulata, $40 \times 3-5$ $\mu \mathrm{m}$. Basidia anguste clavata, tenuitunicata, leviter constricta, (20-)25-30 × 4-5(-6) $\mu \mathrm{m}$, 4 sterigmata. Sporae ellipsoideae, tenuitunicatae, $6-7(-8) \times(2.75-) 3-3.25(-3.5) \mu m$, hyalinae, leves.

Holotypus: Brazil, São Paulo, Ubatuba, Ilha Anchieta, on wood, 17-18 Jan. 1987, Ryvarden 24235 (K (M) 67355. Isotypus: O.

Basidiome resupinate, loosely adnate. Hymenophore strongly raduloid to irpicoid or almost poroid, ochraceous to pale brown, not changing colour in $\mathrm{KOH}$, ridges $0.8-1 \mathrm{~mm}$ high, about $1-2 /$ $\mathrm{mm}$, mostly smooth. Margin indeterminate, revoluble. Subiculum rather thin, concolorous with the hymenophore or more commonly darker. Cords absent. Hyphal system monomitic; subicular hyphae distinct, thick-walled, 5-7 $\mu \mathrm{m}$ wide, often with right angle branching, hyaline to pale yellow, some strongly encrusted with subhyaline crystals; aculeal hyphae similar, hyaline, more or less interwoven; subhymenial hyphae thinwalled or with slight wall thickening, 3-4 $\mu \mathrm{m}$ wide, smooth; all hyphae without clamp-connexions. Cystidia apparently of one type, rare to almost lacking, insignificantly differentiated, thinwalled, at first obclavate, blunt, or more rarely subcapitate, then almost subulate, about $40 \times 3-5 \mu \mathrm{m}$. Basidia narrowly clavate to clavate, thin-walled, slightly constricted, (20-)25-30 × 4-5(-6) $\mu \mathrm{m}$, with four sterigmata and without a basal clampconnexion. Spores ellipsoid, 6-7(-8) × (2.75-)3$3.25(-3.5) \mu \mathrm{m}$, smooth, thin-walled, hyaline.

This species is reminiscent of $P$. magnoliae (Berk. \& Curtis) Burds., but differs by its strongly irpicoid hymenophore, size and shape of the cystidia, and more directly ellipsoid spores. In P. magnoliae the spores are usually subcylindrical, slightly smaller and the cystidia are prominent, almost clavate and 60-100 $\mu \mathrm{m}$ long and 5-10 $\mu \mathrm{m}$ wide.

Phanerochaete tuberculascens Hjortstam sp. nov.
Basidioma resupinatum, effusum, arcte adnatum, circiter 0.1-0.4 mm crassum, plus minus distincte stratosum. Hymenophorum leve, moderate tuberculatum, pallide ochraceum vel rubescens; margine indeterminato vel leviter fibrilloso. Chordae nullae. Subiculum plus minus distinctum, albidum. Systema hyphale monomiticum; hyphae basales distincte, parallele dispositae, leves, tenuitunicatae vel crassiusculae, 5-8 um latae, hyalinae vel plerumque pallide luteolae; fibulae nullae vel rariores. Cystidia absentes. Basidia plus minus clavata, vulgo constricta, 40-50 × 6-7 $\mu \mathrm{m}, 4$ sterigmata. Sporae subglobosae, tenuitunicatae, leves, hyalinae, (5-)5.5-6.5(-7) × $44.75 \mu \mathrm{m}$.

Holotypus: Burundi, T.Muramvya, Teza on wood, 20.XII.1978 J. Rammeloo 6159 (K). Isotypus: O, BR. Paratypi: ditto J. Rammeloo 6160 and from Burundi, Bururi, 4.II.1979 J. Rammeloo 6557 (K, O).

Basidiome resupinate, effused, closely adnate but loosening slightly at the margin, $0.1-0.4 \mathrm{~mm}$ thick, usually stratified. Hymenophore smooth or moderately tuberculate, pale yellowish or (particularly with age or in the herbarium) reddish brown. Margin abrupt or thinning out, not or slightly fibrillose. Subiculum more or less distinct, paler than the fertile part. Cords absent. Hyphal system monomitic; basal hyphae distinct, more or less parallel and densely arranged, smooth, thin-walled or those next to the substratum with slight wall thickening or with slight wall thickening, 5-8 $\mu \mathrm{m}$ wide, hyaline to pale yellowish, without or with occasional clamp-connexions; subhymenial hyphae narrower, 3-5 $\mu \mathrm{m}$ wide, without clamp-connexions. Cystidia absent. Basidia clavate, when fully developed sinuous or constricted, arranged in a rather dense layer, 40-50 × 6-7 $\mu \mathrm{m}$, with four sterigmata and without a basal clamp-connexion. Spores subglobose, smooth, thin-walled (in $\mathrm{KOH}$ slightly thickwalled), hyaline, (5-)5.5-6.5(-7) × $44.75 \mu \mathrm{m}$.

Microscopically this species is reminiscent of P. tuberculata (P. Karst.) Parmasto, but can readily be separated by broader subicular hyphae, denser basidial layer, and slightly broader and subglobose spores. P. tumulosa (P.H.B. Talbot) Hjortstam and P. emplastra (Berk. \& Broome) Hjortstam are also fairly similar, but both have the subicular hyphae more or less intricately woven and narrower spores. 


\section{Key to tropical and subtropical species of Phanerochaete}

1. Hymenophore hydnoid, raduloid or irpicoid

Key 1

1. Hymenophore smooth or rarely grandinioid to tuberculate .. 2

2. Cystidia absent Key 2

2. Cystidia present Key 3

\section{Key 1}

1. Cystidia absent 2

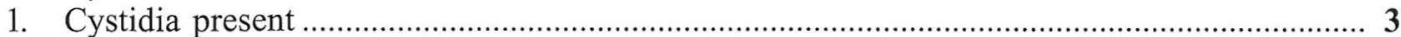

2. Basidiome ceraceous, hymenophore grandinioid, rosy, spores $4-5 \times 3 \mu \mathrm{m}$. Africa (southern and eastern), New Zealand rosea

2. Basidiome hard and brittle, hymenophore hydnoid to more rarely raduloid, tissue dense, hyphae more or less smooth, thin-walled, spores 4.5-6 × 2.5-3.5(-4) $\mu \mathrm{m}$ (type of Radulum subquercinum Henn.). Seems to be pantropical .... subquercina

3. Hymenophore orange or yellowish, aculei almost cylindrical 4

3. Hymenophore otherwise coloured, aculei differently shaped .............................................. 5

4. Hymenophore pale to deep orange, margin and cords reddish orange, spores 4-5 $\times 2-2.5 \mu \mathrm{m}$. USA (Florida), Brazil ?, Cameroon

chrysorhizon

4. Hymenophore yellowish, 5-6 × 3-3.5 $\mu \mathrm{m}$, cords whitish or absent, margin and subiculum white. USA (Florida), Australia?

5. Cystidia subulate, spores $6-8 \times 3-3.5 \mu \mathrm{m}$. Brazil, known only from the type omnivora

5. Cystidia obtuse. irpicoides

6. Aculei $1-2 \mathrm{~mm}$ long, spores $5.5-7 \times 2.5-3 \mu \mathrm{m}$. USA (Florida), Brazil magnoliae

6. Aculei shorter, about $1 \mathrm{~mm}$, spores $4.5-5.5 \times 2-2.5$. Iran and reported from Taiwan aculeata

\section{Key 2}

1. Subiculum tomentose, brownish, hymenophore pale brown, basal hyphae yellowish brown 5-7 $\mu \mathrm{m}$ wide, spores normally 4-6 × 2.5-3.5 $\mu \mathrm{m}$. South America, Taiwan, Australia, New Zealand singularis

1. Subiculum not with a brownish tomentum, mainly whitish, basal hyphae more or less hyaline .... 2

2. Spores ellipsoid to suballantoid and slightly sigmoid, 7-11 $\times 2.3-3.5 \mu \mathrm{m}$, hymenophore pale ochraceous to orange or brownish in old specimens. Hawaii?

2. Spores ellipsoid or cylindric, never sigmoid jose-ferreirae

3. Basal hyphae mainly thick-walled ............................................................................... 4

3. Basal hyphae mainly thin-walled or with slight wall thickening .............................................. 6

4. Hymenophore orange-yellow to yellowish brown, spores 7.5-9.5 $\times$ 4.5-6 $\mu \mathrm{m}$. Canary Islands, known only from the type.....

4. Spores smaller 5

5. Basidiome cracking, hymenophore pale ochraceous to more or less reddish brown, spores 5.5-7 × 3-3.5 $\mu \mathrm{m}$. Sri Lanka, known only from the type.....

5. Basidiome generally not cracking, hymenophore cream, spores 6-8 $\times 3.5-4 \mu \mathrm{m}$. USA (Louisiana) Burdsall (1985), Brazil?, Australia, New Zealand

[5. Spores 5-6 $\times 3.5 \mu \mathrm{m}$. Known only from the type. India ..... Corticium albidocremeum]

6. Subicular hyphae mainly arranged parallel with the substratum .............................................. 7

6. Subicular hyphae more or less intricately woven .......................................................... 8

7. Hymenophore creamish to pale ochraceous, subicular hyphae in a relatively open tissu, cords normally present, spores 5-6.5 × 3-4 $\mu \mathrm{m}$. Cosmopolitan? tuberculata

7. Hymenophore pale yellowish, in the herbarium reddish brown, subicular hyphae in a dense tissue, cords absent, spores (5-)5.5-6.5(-7) × 44.75 $\mu \mathrm{m}$. Africa (Burundi). tuberculascens 
8. Hymenophore slightly greenish in $\mathrm{KOH}$, spores cylindric 5.5-8 $\times 2.25-2.75 \mu \mathrm{m}$. Taiwan, known only from the type. intertexta

8. Hymenophore negative in KOH, spores ellipsoid ........................................................... 9

9. Hymenophore pale reddish brown, cracking, spores $4.5-5.5 \times 3-4.2 \mu \mathrm{m}$ (in the original description). South Africa, known only from the type

tumulosa

9. Hymenophore and spores otherwise .

10

10. Hymenophore pale brown, cracking, subicular hyphae intricately woven, spores 4.5-6 $\times 3-3.5$ (original), (5.5-)6-7.5(-9) $\times 3.5-4.5(-5.5) \mu \mathrm{m}$ (Burdsall 1985). New Zealand, known only from the type

10. Hymenophore not brown, if brownish then spores larger... cordylines

11. Hymenophore sulphurous, yellowish cords present, spores 4-5.5 × 2-2.5 m. Taiwan, known only from the type. lutea

11. Hymenophore and spores otherwise 12

12. Hymenophore yellowish white to greyish orange, spores $6-9 \times 3.5-4.5 \mu \mathrm{m}$. USA (Arizona), Costa Rica xerophila

12. Hymenophore dull yellow, in the herbaria chestnut-brown, spores broadly ellipsoid $6-6.75(-8) \times$ 4-4.5(-5) $\mu \mathrm{m}$. Taiwan

[12.On Citrus from India (Bengal), hymenophore yellowish-white, spores 5.5-7 × 3.5-4.25 $\mu \mathrm{m}$ citri]

\section{Key 3}

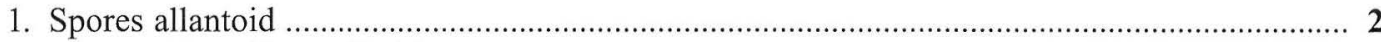

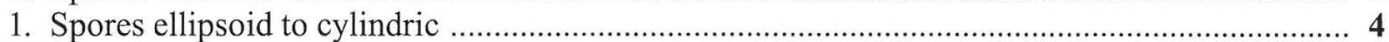

2. Cystidia metuloidal, spores 3.5-4.5 × 1.2-1.5 $\mu \mathrm{m}$. USA (Florida), known only from the type

2. Cystidia cylindric, obtuse, smooth or lightly encrusted

3. Cystidia often septate, lightly encrusted, spores $8.5-14 \times 3.5-5.5 \mu \mathrm{m}$. Brazil cacaina

3. Cystidia not septate, usually smooth, spores narrower, $9-14 \times 2.5-3.5 \mu \mathrm{m}$. USA (Arizona)

4. Wood distinctly discoloured red, hymenophore pale yellow to reddish, subiculum and cords reddish, spores $4.5-6 \times 2.5-3 \mu \mathrm{m}$. Subtropical areas of the northern hemisphere sanguinea

4. Wood never discoloured red, subiculum otherwise, mainly white, but sometimes red or reddish brown

5. Spores (8-)9-12 × 4-6 $\mu \mathrm{m}$, cystidia subulate or sometimes obtuse, normally smooth. Madeira, Morocco, Australia

martelliana

5. Spores smaller 6

6. Hymenophore yellowish to orange, cords absent, cystidia of two kinds 1) heavily encrusted 2) nearly smooth, both cystidia with orange crystals, spores $5-5.5 \times 2.5-3 \mu \mathrm{m}$. New Zealand, known only from the type

luteoaurantiaca

6. Not with this combination of character 7

7. Cystidia generally strongly encrusted, sometimes metuloidal …........................................... 8

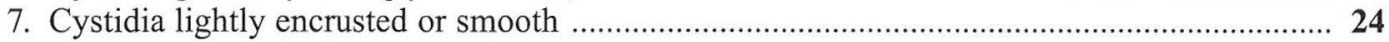

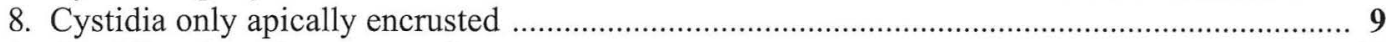

8. Cystidia otherwise encrusted ............................................................................................... 10

9. Hymenophore usually whitish, creamish to pale ochraceous, spores 4-5.5 × 2-2.5 $\mu \mathrm{m}$ Borneo (type), Hawaii, Brazil, Colombia australis

9. Hymenophore ochraceous to pale red, spores 5-6×2.75-3 $\mu \mathrm{m}$. Sri Lanka flavocarnea

10. Hymenophore and cords red or purple in $\mathrm{KOH}$, subiculum yellowish or yellowish brown, subicular hyphae 6-8(-10) $\mu \mathrm{m}$ wide, cystidia 40-100 $\mu \mathrm{m}$ long, spores $(3.5-) 4-5.5(-6.5) \times 2-2.5(-3) \mu \mathrm{m}$. Pantropical. (P. borneensis Jülich, hyphae as in P. radicata, but generally with thicker walls, cystidia $20-35 \mu \mathrm{m}$ long) radicata 
[10. Hymenophore sulphur-coloured to mustard yellow, and with conspicuous chrome-yellow cords. Described from Brazil, but insufficiently known, holotype poor.

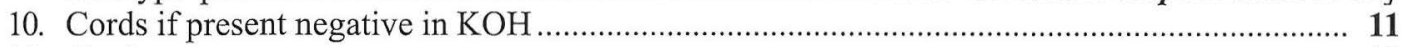

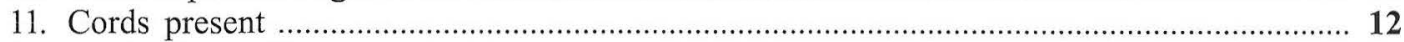

11. Cords absent, if present then hymenophore strongly pilose ........................................... 14

12. Hymenophore light yellow to light orange, red in $\mathrm{KOH}$, spores $4.5-6 \times 2.5-3 \mu \mathrm{m}$. USA (Florida and Louisiana)

salmoneolutea

12. Hymenophore otherwise coloured, negative or maybe pale brown in $\mathrm{KOH}$......................... 13

13. Hymenophore whitish to pale ochcareous, in the herbaria sometimes orange coloured, cystidia subulate, strongly encrusted, but apically nude, spores 5-7 $\times 2.5-3 \mu \mathrm{m}$. Distribution uncertain, but reported by several authors, mainly as Peniophora (Phanerochaete) affinis ............. laevis

13. Hymenophore pale yellow to pale brownish yellow, sometimes pale brown in $\mathrm{KOH}$, cystidia more or less obtuse, normally encrusted throughout, spores 4-5.5 × 2.75-3.5 $\mu \mathrm{m}$. Jamaica, Puerto Rico, Maxico, Panama, Brazil .............................................................................................. flava

14. Cystidia subulate, with a nude apex ............................................................................... laevis

14. Cystidia otherwise encrusted, subulate to obtuse .............................................................. 15

15. Hymenophore usually vinaceous red to brownish orange, strongly pilose by protruding cystidia, spores 5.5-7 × 2.5-3.5 $\mu \mathrm{m}$. Jamaica, Morocco, Canary Islands ........................................ velutina

15. Hymenophore otherwise coloured, not or less pilose ….................................................... 16

16. Hymenophore yellowish to pale olivaceous, greenish in $\mathrm{KOH}$, spores $4.8-6.2 \times 3.3-4.3 \mu \mathrm{m}$. Taiwan, known only from the type

subglobosa

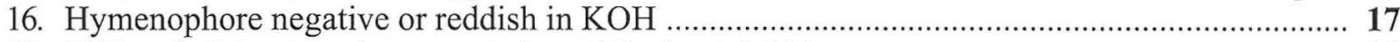

17. With subulate strongly encrusted cystidia (metuloids) .............................................. 18

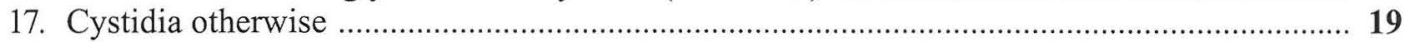

18. Hymenophore creamish to greyish-yellow, basal hyphae thick-walled, cystidia often more than 10-12 $\mu \mathrm{m}$ wide, spores 5-7 × 2.5-3.5 $\mu \mathrm{m}$. Jamaica, USA (Florida), Bermuda, Brazil? (Phlebiopsis?).

hiulca

18. Hymenophore pale ochraceous with a brownish margin, basal hyphae mainly thin-walled, cystidia somewhat slender, spores 6-8 $\times 3-3.5 \mu \mathrm{m}$. New Zealand, known only from the type. A species of Phlebiopsis?

areolata

19. Cystidia 50-100 $\mu \mathrm{m}$ long, lightly or more heavily encrusted ................................................. 20

19. Cystidia generally shorter, $30-50 \mu \mathrm{m}$ long, metuloidal but obtuse ....................................... 22

20. Subicular hyphae thin-walled, cystidia generally up to $70 \mu \mathrm{m}$ long, obtuse and strongly encrusted, spores 5-7 $\times 2.5-3 \mu \mathrm{m}$. Mexico, known only from the type? ....................................... exigua

20. Subicular hyphae thick-walled.

21. Basidiome usually firm membranous, cystidia smooth or encrusted, basal hyphae thick-walled, rigid, spores 5-7.5 × 2.5-3.5 $\mu \mathrm{m}$. Cosmopolitan, but variable.

sordida

21. Basidiome rather soft, creamish to light buff, cystidia strongly encrusted, hyphae thin to firmwalled, spores 5.5-6 $\times 2.5-3 \mu \mathrm{m}$. Hawaii, known only from the type ......................... mauiensis

22. Spores cylindric and often suballantoid, 8-12 × 3-3.5 $\mu \mathrm{m}$. Africa (South Africa, Ethiopia, Kenya).

arenata

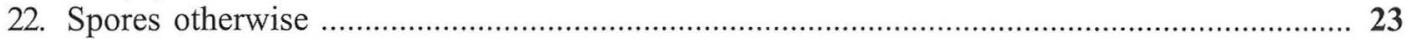

23. Cystidia 5-6 $\mu \mathrm{m}$ wide, spores 5.5-6.5 $\times 3-3.5 \mu \mathrm{m}$ Mexico, USA (Florida), Brazil, Colombia .... exilis

23. Cystidia $7(-10) \mu \mathrm{m}$ wide, spores $6-6.5 \times 3-4 \mu \mathrm{m}$. Paraguay, Brazil .............................. incrustans

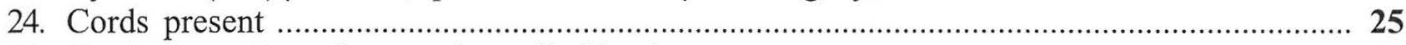

24. Cords absent (margin sometimes fibrillose) ............................................................. 27

25. Cords bright red in $\mathrm{KOH}$, spores 4.5-6 × 2-2.5 $\mu \mathrm{m}$ (Burt $4-4.5 \times 2-2.5 \mu \mathrm{m}$ ). USA (Florida), Jamaica, Argentina, Brazil, Australia ....................................................................................... burtii

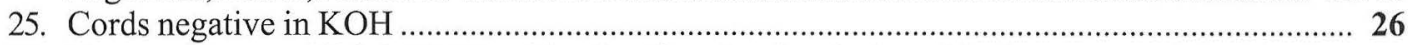

26. Hymenophore reddish in $\mathrm{KOH}$, with arboriform hyphae in the subiculum, spores $4.5-5.5 \times 2.5 \mu \mathrm{m}$. Brazil. 
26. Hymenophore greenish in $\mathrm{KOH}$, without arboriform hyphae, spores $4.5-6 \times 2-3 \mu \mathrm{m}$. Brazil.

carnosa

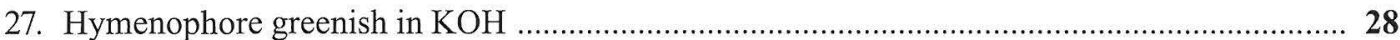

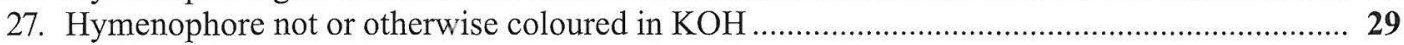

28 Hymenophore dull yellow, spores $4.5-6 \times 2-3 \mu \mathrm{m}$. ................................................ carnosa

28. Hymenophore ivory-yellow or white, spores 3.8-4.5 $\times 1.8-2.3 \mu \mathrm{m}$. Taiwan, known only from

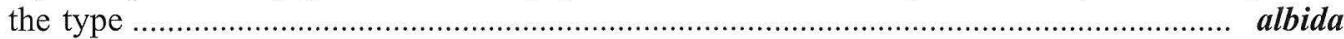

29. Subicular hyphae producing globose vegetative cells, spores $5.5-7(-8) \times 3-3.5 \mu \mathrm{m}$. Puerto Rico, Taiwan sacchari

29. Subicular hyphae lacking such globose cells 30

30. Cystidia tubular, 60-150 $\mu \mathrm{m}$ long, thin-walled, spores 5.5-7 $\times 3-4$. Iran sub Phanerochaete macrocystidiata Hallenb.). chrysosporium

30. Cystidia otherwise 31

31. With arboriform hyphae in the subiculum, cystidia mainly subulate, often few, spores 5.5-6.5 $\times$ 3-3.5 $\mu \mathrm{m}$. Taiwan.... ericina

31. Without arboriform hyphae, cystidia subulate or obtuse 32

32. Subicular hyphae thin-walled or with a slight wall thickening, spores $6.25-8 \times 3-3.25 \mu \mathrm{m}$. Taiwan .

leptoderma

32. Subicular hyphae thick-walled

33. Spores $5-7.5 \times 2.5-3.5 \mu \mathrm{m}$ 33

33. Spores $6.5-8.25 \times 3.5-4.5 \mu \mathrm{m}$ sordida taiwaniana

\section{Checklist}

Phanerochaete aculeata Hallenb., Iran. J. Pl. Path. 14: 62 (1978). Type: Iran, 12.VII.1976 Hallenberg 1737 (GB).

$\boldsymbol{P}$. affinis (Burt) Parmasto, Conspectus syst. corticiacearum: (Tartu) p. 84 (1968). Basionym: Peniophora affinis Burt, Ann. Mo. Bot. Gard. 12: 266 (1926). Type: USA, Vermont, 14.IX.1900 E.A. Burt (BPI). = Phanerochaete laevis.

P. alba H. Lin \& Z.C. Chen, Taiwania 35: 97 (1990). Type: Taiwan, S. Lin (NTU-3407). According to the description and illustration, apparently a species of Phlebiopsis.

P. albida Sheng H. Wu, Acta Bot. Fenn. 142: 39 (1990). Type: Taiwan, on culm of Poaceae, 9.IX.1988 Wu 880909-61 (H).

Corticium albidocremeum Rehill \& B.K. Bakshi, Indian Forest Bull. 242: 11 (1965). Type: India, on burnt bamboo, 20.I.1950 K. Bagchee 5128 (K). = ?Phanerochaete sordida .

P. allantospora Burds. \& Gilb., Mycologia 66: 780 (1974). Type: USA, Arizona, on Platanus wrightii, 23.IX.1971 R. L. Gilbertson 10478 (CFMR).

P. andreae Burds. et al. Mycotaxon 54: 296 (1995). Type: Canary Islands, 5.XII.1987 (TFC).

P. arenata (P.H.B. Talbot) Jülich, Persoonia 10: 334 (1979). Basionym: Peniophora arenata P. H.
B. Talbot, Bothalia 4: 944 (1948). Type: South Africa, Natal, 1934 W.G. Rump 34 (K).

P. areolata (G. Cunn.) Hjortstam \& Ryvarden, Synopsis Fungorum (Oslo) 4: 59 (1990). Basionym: Lopharia areolata G. Cunn., N.Z. Dept. sci. industr. Res. Bull. 145: 331 (1963). Type: New Zealand, 5100 (P.D.D.).

P. australis Jülich, J. Linn. Soc. Bot. 81: 43 (1980). Type: Borneo, Sarawak, 14.III. 1978 Jülich 78-1868(L).

P. borneensis Jülich, Journ. Linn. Soc. Bot. 81: 43 (1980). Type: Borneo, Sara-wak. 16 III. 1978 Jülich 78-2157 (L). Nakasone et al. (1994) accepted this as an independent species.

P. brunnea Sheng H. Wu, Acta Bot. Fenn. 142: 42 (1990). Type: Taiwan, Nantou, 700 m, on twig of angiosperm, 25.X.1988 Wu 881025-23 (H). = Phanerochaete singularis.

P. burtii (Romell) Parmasto, Eesti NSV Tead. Akad. Toim. Biol. 16: 338 (1967). Basionym: Peniophora burtii Romell, Burt Ann. Mo. Bot. Gard. 12: 278 (1926). Type: USA, Ohio, 1898 Lloyd 3823 (S).

P. bubalina Burds., Mycol. Memoir 10: 44 (1985). Type: Canary Islands, Tenerife, 8.I.1974 L. Ryvarden $12356(\mathrm{O})$. = Hyphodermella corrugata (Fr.) J. Erikss. \& Ryvarden 
P. cacaina (Bourdot \& Galzin) Burds. \& Gilb., Mycologia 66: 781 (1974). Basionym: Peniophora cacaina Bourdot \& Galzin, Bull. Soc. Mycol. France 28: 397 (1913). Type: France, Aveyron, Febr. 1908 Galzin 2795, Bourdot 5473.

P. cana (Burt) Burdsall, Mycol. Memoir 10: 50 (1985). Basionym: Peniophora cana Burt, Ann. Mo. Bot. Gard. 12: 227 (1926). Type: USA, Florida, March 1923 Murrill 82 (BPI).

P. carnosa (Burt) Parmasto, Eesti NSV Tead. Akad. Toim. Biol. 16: 388 (1967). Basionym Peniophora carnosa Burt, Ann. Mo. Bot. Gard. 12: 325 (1926). Type: USA, New York, on Juniperus nana, 19.IX.1901 C.H. Peck, $H M B G$ 56019 (BPI).

P. chordalis (Höhn. \& Litsch.) Park.-Rhodes, Ann. Bot., London N.S. 20: 258 (1956). Basionym: Peniophora chordalis Höhn \& Litsch., Sitzber. Akad. Wiss. Wien, Math.-nat. Kl. 115: 1598 (1906). Type: Austria, Niederösterreich, Wechsel, 2.VI.1906. = Xenasma pruinosum (Pat.) Donk

P. chrysorhizon (Torrey) Bud. \& Gilb., Southw. Natur. 17: 417 (1973). Basionym: Hydnum chrysorhizon Torrey, in Eaton Manual Bot. 309 (1822). Type: USA, Steward 237 in Herb. Torrey (NY).

P. chrysosporium Burds., Mycotaxon 1: 124 (1974). Type: USA, Arizona, 25. VIII.1971 Burdsall 6251 (CFMR). Anamorph: Sporotrichum pruinosum Gilman \& Abbot, see further Stalpers (1984).

P. citri A.B. De, Mycotaxon 42: 29 (1991). Type: India, West Bengal, on Citrus medica, 21.X.1988 A.B.De (BRCMH C-881, portion K).

P. commixtoides $\mathrm{H}$. Lin \& Z.C. Chen, Taiwania 35: 99 (1990). Type: Taiwan, S. Lin (NTU-3644). According to the description and illustration this seems to be a species of Dendrothele.

P. cordylines (G. Cunn.) Burds., Mycol. Memoir 10: 63 (30.I. 1985). Basionym: Corticium cordylines G. Cunn., Trans. R. Soc. New Zeal. 82: 323 (1954). Type: New Zealand, on Cordyline australis, G.T. Baytes 7405. (P.D.D.).

P. corymbata (G. Cunn.) Burds., Mycol. Memoir 10: 65 (1985). Corticium corymbata G. Cunn. Trans. R. Soc. New Zeal. 82: 324 (1954). Type: New Zealand, on Brachyglottis repanda, May 1952 G.H. Cunningham 11474. (P.D.D.)

P. crassa (Lév.) Burds. Mycol. Memoir 10: 67 (1985). Basionym: Thelephora crassa Lév. Ann. Sci. Nat. Bot. ser.III, 2: 209 (1844). Type: Vietnam (BPI). = Porostereum crassum (Lév.) Hjortstam \& Ryvarden.
P. cremea (Bres.) Parmasto, Conspectus syst. corticiacearum: (Tartu) p. 84 (1968). Basionym: Corticium cremeum Bres., Fungi Trid. 2: 63 (1898). Type: Italy, Trento, in ramis Sorbi aucupariae, Aug 1893 Bresadola 556 (S). = Phanerochaete sordida.

P. cumulodentata (Nikol.) Parmasto, Conspectus syst. corticiacearum: (Tartu) p. 83 (1968). Basionym: Radulum cumulodentatum Nikol., in Fl. Plant. Crypt. 6: 87 (1961). = Nom. rej., not validly published

Radulum cumulodentatum Nikol., Mikol. Fitopatol. 4: 477 (1970). Type: Russia, Orel Reg., on Sorbus aucuparia, 27 Aug 1916 A.S. Bondarzew (LE). = Phanerochaete magnoliae .

Corticium decolorans P. Karst., Bidr. Känned. Finl. Nat. Folk 37: 144 (1882). Type: Finland, Mustiala, Salix, Oct 1879 P.A. Karsten $1405(\mathrm{H})$. = Phanerochaete velutina.

P. emplastra (Berk. \& Broome) Hjortstam, Kew Bull. 44: 306 (1989). Basionym: Corticium emplastrum Berk. \& Broome J. Linn. Soc. Bot. 14: 70 (1875). Type: Sri Lanka, Central Prov., Dec 1868, no. 985 (K).

P. ericina (Bourdot) J. Erikss. \& Ryvarden, Corticiaceae North Eur. 5: 1011 (1978). Basionym: Peniophora ericina Bourdot, Rev. Sci. Bourbonn. 23: 14 (1910). Type: France, Aveyron, on Erica sp., 9.IV.1910 Galzin 5442, Bourd. 8632, Lloyd 44574 (BPI).

P. exigua (Burt) Nakasone et al., Mycologia 90: 134 (1998). Basionym: Peniophora exigua Burt, Ann. Mo. Bot. Gard. 12: 224 (1926). Type: Mexico, Guernavaca, Murrill 377 (BPI).

P. exilis (Burt) Burds., Mycol. Memoir 10: 74 (1985). Basionym: Peniophora exilis Burt, Ann. Mo. Bot. Gard. 12: 239 (1926). Type: Mexico, Orizaba, 10-14.I.1910 Murrill 757 (BPI).

P. filamentosa (Berk. \& M.A. Curtis) Burds., in Parker \& Roane, Distr. Hist. Biota southern. Appalachians IV. Algae and Fungi p. 278 (1976). Basionym: Corticium filamentosum Berk. \& M.A. Curtis, Grevillea 1: 178 (1873). Type: USA, Alabama, Peters 6119 (K).

P. flabelliradiata J. Erikss. \& Hjortstam Corticiaceae North Eur. 6: 1073 (1981). Type: Norway, Akershus, 28.IX.1978 Ryvarden 17494. = Leifia flabelliradiata (J. Erikss. \& Hjortstam) Ginns.

P. flava (Burt) Nakasone et al. Mycologia 90: 132 (1998). Basionym: Coniophora flava Burt, Ann. Mo. Bot. Gard. 4: 261 (1917). Type: Jamaica, W.A. \& E.L. Murrill 1089 (NY). 
P. flavidoalba (Cooke) S.S. Rattan, Bibl. Mycol. 60: 262 (1977). Basionym: Peniophora flavidoalba Cooke, Grevillea 8: 21 (1879). Type: USA, Georgia, Darien, on Myrica cerifera, Ravenel 2529, Fung. amer. Exs. 226 (NY). = Phlebiopsis.

P. flavocarnea (Petch) Hjortstam, Mycotaxon 54: 189 (1995). Basionym: Corticium flavocarneum Petch, Ann. R. Bot. Gard., Peradeniya 9: 288 (1925). Type: Sri Lanka, Hakgala, April 1915, No. $4672(\mathrm{~K})$.

P. fuscomarginata (Burt) Gilb., J. Arizona Acad. Sci. 7: 135 (1972). Basionym: Peniophora fuscomarginata Burt, Ann. Mo. Bot. Gard. 12: 335 (1926). Type: USA, Louisiana, St. Martinville, 15.VII.1897 A.B. Langlois 100 (FH). = Porostereum fuscomarginatum (Burt) Hjortstam.

P. gigantea (Fr. : Fr.) S. S: Rattan, Bibl. Mycol. 60: 260 (1977). Basionym: Thelephora gigantea Fr. : Fr., Syst. Mycol. 1: 448 (1821). Type: Sweden, Femsjö, E. Fries (UPS). = Phlebiopsis.

P. globosa H. Lin \& Z.C. Chen, Taiwania 35: 100 (1990). Type: Taiwan, S. Lin (NTU 2427). According to the description and illustration this seems to be a species of Candelabrochaete Boidin.

P. himalayensis (Dhingra) Sheng $\mathrm{H}$. Wu, Ann. Bot. Fenn. 142: 45 (1990). Basionym: Phlebiopsis himalayensis Dhingra, Nova Hedwigia 44: 222 (1987). Type: India, West Bengal, 9.VIII.1980 G.S. Dhingra 19202 (GB, isotype). = Phlebiopsis.

P. hiulca (Burt) Welden, Mycotaxon 10: 441 (1980). Basionym: Peniophora hiulca Burt, Ann. Mo. Bot. Gard. 12: 272 (NY). Type: Jamaica, Castleton Gardens, W.A. \& E.L. Murrill 71 (NY).

Peniophora hydnoides Cooke \& Massee, Grevillea 16: 77 (1888). Type: England, Carlisle, Nov $1987(\mathrm{~K}) .=$ ?Scopuloides rimosa (Cooke) Jülich.

P. incrustans (Speg.) Rajchenb. \& Wright, Mycologia 79: 255 (1987). Basionym: Odontia incrustans Speg. An. Soc. Cient. Argent. 90: 168 (1921). Type: Paraguay?, Asuncion, on Citrus aurantium, X-1919 (LPS).

P. insolita Burds. \& Nakasone, Mycologia 73 (3): 467 (1981). Type: USA, Florida, Liquidambar styraciflua, 26.VII.1977 H.H. Burdsall 9561 (CFMR). = Candelabrochaete langloisii (Pat.) Boidin.

P. intertexta Sheng $\mathrm{H}$. Wu, Acta Bot. Fenn. 142: 45 (1990). Type: Taiwan, Wu 26.VII.1988 No. 880726-50(H).
P. jose-ferreirae (D.A. Reid) D.A. Reid, Acta Bot. Croat. 34: 135 (1975). Basionym: Corticium jose-ferreirae D.A. Reid, Rev. Biol. 5: 140 (1965). Type: Portugal, 10.V.1964 D.A. Reid (K).

P. karstenii (Bres.) P. Karst., Med. Soc. Fauna Fl. fenn. 1: 162 (1889). Basionym: Stereum karstenii Bres., Atti I. R. Accad. Agiati ser. III, 3: 109 (1897). Type: Hungary, A. Kmet (S?). = Dacryobolus karstenii (Bres.) Oberw.

P. laevis (Pers. : Fr.) J. Erikss. \& Ryvarden, Corticiaceae North Eur. 5: 1007 (1978). Basionym: Thelephora laevis Pers. : Fr., Syst. Mycol. 1: 451 (1821). Type: Corticium laeve Fr. Herb. M.J. Berkeley, $1879(\mathrm{~K})$.

P. leprosa (Bourdot \& Galzin) Jülich, Persoonia 10: 334 (1979). Basionym: Peniophora radicata ssp. leprosa Bourdot \& Galzin, Bull. Soc. Mycol. France 28: 394 (1913). Type: France, Aveyron, on Fagus sp., 9.V.1908 Galzin 3257, Bourdot $5637(\mathrm{PC}) .=$ Phanerochaete velutina.

P. leptoderma Sheng $\mathrm{H}$. Wu, Acta Bot. Fenn. 142: 45 (1990). Type: Taiwan, Hsinchu, $1000 \mathrm{~m}$, on branch of angiosperm, 5.IV.1988 Wu 880405-13 (TAI).

P. lutea (Sheng H. Wu) Hjortstam, Mycotaxon 54: 189 (1995). Basionym: Efibula lutea Sheng. H. Wu, Acta Bot. Fenn. 142: 23 (1990). Type: Taiwan, Taipei, 17.IV. 1988 Wu 880417-5 (H).

P. luteoaurantiaca (Wakef.) Burds., Mycol. Memoir 10: 93 (1985). Basionym: Corticium luteoaurantiacum Wakef., Kew Bull. Misc. Inf. 1915: 372. Type: New Zealand, 1914 W.N. Cheeseman $17455(\mathrm{~K})$.

P. macrocystidiata Hallenb., Iran. J. Pl. Path. 14: 65 (1978). Type: Iran, Gorgan, Hallenberg 1618. = Phanerochaete chrysosporium Burds.

P. macrospora (Bres.) Parmasto, Conspectus syst. corticiacearum: (Tartu) p.84 (1968). Basionym: Peniophora macrospora Bres. in Bourdot \& Galzin, Bull. Soc. Mycol. France 28: 396 (1913). Type: France, Allier, 27.VII.1905 Bourdot 4024 (PC).

P. magnoliae (Berk. \& M.A. Curtis) Burds., Mycol. Memoir 10: 95 (1985). Basionym: Radulum magnoliae Berk. \& M.A. Curtis, Hooker's J. Bot. 1: 236 (1949). Type: USA, South Carolina on Magnolia glauca, May Curtis 1097 (K).

P. mauiensis Gilb. \& Adask., Mycotaxon 49: 384 (1993). Type: Hawaii, on Eugenia jambos, J.E. Adaskaveg 1462 (BPI).

P. monomitica (G. Cunn.) Sheng H. Wu \& Popoff, Mycotaxon 54: 167 (1995). Basionym: 
Duportella monomitica G. Cunn., Trans. R. Soc. New Zeal. 85: 98 (1957). Type: New Zealand, Auckland, on Leptospermum scoparium 16644 (P.D.D.). $=$ Porostereum monomiticum (G. Cunn.) Hjortstam \& Ryvarden.

P. omnivora (Shear) Burds. \& Nakasone, Mycotaxon 7: 17 (1978). Basionym: Hydnum omnivorum Shear, J. Agric. Res. 30: 476 (1925). Type: USA, Texas, Sep 1903 Shear 5267 (BPI).

P. pallida Parmasto Eesti NSV Tead. Akad. Toim. Biol. 16: 388, (1967). Type: Russia 28.IX.1961 Parmasto 14686.

P. parmastoi Sheng H. Wu, Acta Bot. Fenn. 142: 49 (1990). Type: Taiwan, Wu No. 880313$6(\mathrm{TAI}) .=$ Phanerochaete sacchari .

P. pelliculosa (P.H.B. Talbot) Jülich, Persoonia 10: 334 (1979). Basionym: Peniophora pelliculosa P.H.B. Talbot, Bothalia 6: 63 (1951). Type: Africa, Krantzkop, Dec.1935 W.G. Rump No. 450 (K). Seems to be the same as Phanerochaete sordida.

P. phosphorescens (Burt) Welden, Mycotaxon 10: 445 (1980). Basionym: Peniophora phosophorescens Burt, Ann. Mo. Bot. Gard. 12: 273 (1926). Type: Jamaica, A.E. Wright 1909 (FH). = Phanerochaete velutina, fide Burdsall (1985).

P. pruni (Lasch) S.S. Rattan, Bibl. Mycol. 60: 258 (1977). =Hyphodontia pruni (Lasch) Svrcek.

P. radicata (Henn.) Nakasone et al., Sydowia, Ann. Mycol. Ser. II, 46: 46 (1994). Basionym: Corticium radicatum Henn., Engler Pflanzenwelt Ost-Afrikas, Lieferung 1, Theil C., p. 54 (1895). Type: Africa, Usambara, Nderema, im Urwald auf morschen Baumstümpfen, Holst 2314 (S, isotype).

P. radulans Hallenb., Iran. J. Pl. Path. 14: 67 (1978). Type: Iran, 3.VII.1976 L. \& N. Hallenberg \& D. Ershad (GB). = Phanerochaete subquercina.

P. raduloides J. Erikss. \& Ryvarden, Corticiaceae North Eur. 5: 1015 (1978). Type: Finland, on Corylus avellana, 26.IX.1970 T. Niemelä (H). = Phanerochaete magnoliae.

P. ravenelii (Cooke) Burds. Mycol. Memoir 10: 104 (1985). Basionym: Peniophora ravenelii Cooke, Grevillea 8: 21 (1879). Type: USA, South Carolina, Ravenel. (K). A species of Phlebiopsis.

P. rimosa (Cooke) Burds., Mycol. Memoir 10: 107 (1985). Basionym: Peniophora rimosa Cooke, Grevillea 9: 94 (1881). Type: Coed Coch, on bark, Oct. $1880(\mathrm{~K}) .=$ Scopuloides rimosa (Cooke) Jülich
P. rosea (Henn.) Buchanan \& Hood, New Zeal. J. Bot. 30: 105 (1992). Basionym: Grandinia rosea Henn., Engl. Bot. Jahrb. 38: 108 (1907). Type: Tanzania, Ost-Usambara, Aug. 1903 Eichelbaum 72 $C(\mathrm{~S})$.

P. sacchari (Burt) Burds., Mycol. Memoir 10: 113 (1985). Basionym: Peniophora sacchari Burt, Ann. Mo. Bot. Gard. 12: 328 (1926). Type: Puerto Rico, on Saccharum officinarum, J.A. Stevenson 1204 (BPI).

P. sacrata (G. Cunn.) Taylor, New Zeal. J. Agric. Res. 24: 373 (1981). Basionym: Penophora sacrata G. Cunn., Trans. R. Soc. New Zeal. 83: 274 (1955). Type: New Zealand, Leptospermum scoparium, Jan. 1953 J.D. Atkinson 11845 (P.D.D.). = Gloeocystidiellum sacratum (G. Cunn.) Stalpers \& P.K. Buchanan.

P. salmoneolutea Burds. \& Gilb. Mycologia 66: 787 (1974). Type: USA, Florida, on Carya sp., 14.VII.1972 (BPI).

P. salmonicolor (Berk. \& Broome) Jülich Persoonia 8: 294 (1975). Basionym: Corticium salmonicolor Berk. \& Broome, J. Linn. Soc. Bot. 14: 71 (1873). Type: Sri Lanka, Peradenia, 4.XI.1867, Herb. Berk. $3968(\mathrm{~K})$. = A species of Erythricium J. Erikss. \& Hjortstam and is comparativley similar to E. laetum (P. Karst.) J. Erikss. \& Hjortstam.

P. sanguinea (Fr.) Pouzar, Ceská Mykol. 27: 26 (1973). Basionym: Thelephora sanguinea Fr., Elench. fung. 1: 203 (1828). Type: Sweden (?), det. by E. Fries (E).

P. septocystidia (Burt) J. Erikss. \& Ryvarden, Corticiaceae North Eur. 5: 1021 (1978). Basionym: Peniophora septocystidia Burt, Ann. Mo. Bot. Gard. 12: 260 (1926).Type: Jamaica, W.A. Murrill \& W. Harris 860, 12-14.I. 1909 (BPI). = Candelabrochaete septocystidia (Burt) Burds.

P. singularis (G. Cunn.) Burds., Mycol. Memoir 10: 121 (1985). Basionym: Corticium singulare G. Cunn., Trans. R. Soc. New Zeal. 82: 325 (1954). Type: New Zealand, on Litsea calicaris, 17.VI.1950 J.M. Dingley 19589 (P.D.D.).

P. sordida (P. Karst.) J. Erikss. \& Ryvarden, Corticiaceae North Eur. 5: 1023 (1978). Basionym: Corticium sordidum P. Karst., Medd. Soc. Fauna Fl. Fenn. 9: 65 (1882). Type: Fennia, Mustiala, 21.X.1865 P.A. Karsten $1512(\mathrm{H})$.

P. stereoides Sheng H. Wu, Mycotaxon 54: 168 (1995). Type: Taiwan, Taipei, on branch of angiosperm, 25.VII.1991 (N.M.N.S.). Seemingly a species of Porostereum and is reminiscent of $P$. 
monomiticum (G. Cunn.) Hjortstam \& Ryvarden. and P. perplexum (D.A. Reid) Hjortstam \& Ryvarden.

P. subceracea (Burt) Burds., Mycol. Memoir 10: 128 (1985). Basionym: Corticium subceraceum Burt, Ann. Mo. Bot. Gard. 13: 239 (1926). Type: USA, Pennsylvania, Trexlertown, $W$. Herbst 76 (NY).

P. subglobosa Sheng H. Wu, Acta Bot. Fenn. 142: 49 (1990). Type: Taiwan, Taipei, on bark of living Melaleuca leucadendron 18.IX.1987 Wu $870918(\mathrm{H})$.

P. subiculosa (Burt) Burds., Mycol. Memoir 10: 130 (1985). Basionym: Peniophora subiculosa Burt, Ann. Mo. Bot. Gard. 12: 259 (1926). Type: Mexico, Cuernavaca, Tepeite River, 28.XII.1909 Murrill 396 (BPI). = Phanerochaete flava, fide Nakasone et al. (1998)

P. subquercina (Henn.) Hjortstam, Windahlia 17: 58 (1987). Basionym: Radulum subquercinum Henn., Monsunia I, Fungi 2: 46 (1899). Type: Java, Salek, 11.IX.1897 E. Nyman (S).

P. sulphurina (P. Karst.) Bud. \& Gilb., The Southwest. Naturalist 17: 417 (1973). Basionym: Tomentella sulphurina P. Karst., Bidr. Känned. Finl. Nat. Folk 48: 420 (1889). Type: Finland, Jalasjärvi, P.A. Karsten $(\mathrm{H})$. = Ceraceomyces sulphurinus (P. Karst.) J. Erikss. \& Ryvarden.

Corticium sulphurosum Bres., Annls mycol. (Berlin) 18: 47 (1920). Type: Brazil, Bahia, Torrend 59 (S).

P. taiwaniana Sheng H. Wu, Acta Bot. Fenn. 142: 52 (1990). Type: Taiwan, Miaoli, $1900 \mathrm{~m}$, on fallen branch of angiosperm, 24.VIII.1988 Wu 880824-17 (H).

P. tropica (Sheng H. Wu) Hjortstam Mycotaxon 54: 189 (1995). Basionym: Efibula tropica Sheng. H. Wu., Acta Bot. Fenn. 142: 25 (1990). Type: Taiwan, Taipei, on Ficus virgata, 3.XII.1987 Wu 871203 (TAI). Generic type of Efibula Sheng H. Wu.

P. tuberculata (P. Karst.) Parmasto, Conspectus syst. corticiacearum: (Tartu) p. 83 (1968). Ba- sionym: Corticium tuberculatum P. Karst., Hedwigia 35: 45 (1896). Type: Finland, Mustiala, 7.X.1895 P.A. Karsten $1503(\mathrm{H})$.

P. tumulosa (P.H.B. Talbot) Hjortstam, Mycotaxon 54: 189 (1995). Basionym: Corticium tumulosum P.H.B. Talbot, Bothalia 4: 941 (1948). Type: South Africa, Pretoria, 27.IV.1937 K.A. Landsdell 28897 (K).

P. velutina (DC. : Fr.) P. Karst., Krit. Öfvers. Finl. Basidsv. Tillägg 3: 33 (1898). Basionym: Thelephora velutina DC. : Fr., Elenchus fung. 1: 203 (1828). Type: See Burdsall (1985).

P. viticola (Schwein. : Fr.) Parmasto, Eesti NSV Tead. Akad. Toim. Biol. 16 (4): 389 (1967). Basionym: Thelephora viticola Schwein. : Fr., Elench. fung. 1: 205 (1828). Type: 691-87-Syn. Fung. Salem-Beth. $(\mathrm{PH}) .=$ Closely related to Globulicium Hjortstam?

P. xerophila Burds., Mycol. Memoir 10: 141 (1985). Type: USA, Arizona, on Prosopis velutina, 26.II.1971 R.L. Gilbertson 10082 (CFMR).

Acknowledgement: I wish to express my appreciation to Dr. Peter Roberts, Royal Botanic Gardens, Kew who revised the English language and suggested improvements.

\section{References}

Burdsall, H. H. 1985: A contribution to the taxonomy of the genus Phanerochaete (Corticiaceae, Aphyllophorales). - Mycologia Mem. 10: 1-165.

Hjortstam, K. and Larsson, K. H. 1995: Annotated check-list to genera and species of corticioid fungi (Aphyllophorales, basidiomycotina) with special regards to tropical and subtropical species. - Windahlia 21: $1-75$.

Nakasone, K. K., Bergman, C. R. and Burdsall H. H. 1994: Phanerochaete filamentosa - Corticium radicatum species complex in North America. - Sydowia 46: 44-62.

Nakasone, K. K., Burdsall, H. H. and Lodge, D. J. 1998: Phanerochaete flava in Puerto Rico. - Mycologia 90: $132-135$.

Stalpers, J. A. 1984: A revision of the genus Sporotrichum. - Stud. Mycol. 24: 1-105. 